\title{
Behavioural activation for depression
}

\author{
David Veale
}

\begin{abstract}
A formal therapy for depression, behavioural activation focuses on activity scheduling to encourage patients to approach activities that they are avoiding and on analysing the function of cognitive processes (e.g. rumination) that serve as a form of avoidance. Patients are thus refocused on their goals and valued directions in life. The main advantage of behavioural activation over traditional cognitive-behavioural therapy for depression is that it may be easier to train staff in it and it can be used in both in-patient and out-patient settings. This article describes the theory and rationale of behavioural activation, its evidence base and how to develop a formulation that guides the strategy.
\end{abstract}

Over three decades ago, Ferster (1973) developed a model of depression based on learning theory: it stated that when people become depressed, many of their activities function as avoidance and escape from aversive thoughts, feelings or external situations. Depression therefore occurs when a person develops a narrow repertoire of passive behaviour and efficiently avoids aversive stimuli. As a consequence, someone with depression engages less frequently in pleasant or satisfying activities and obtains less positive reinforcement than someone without depression. Lewinsohn et al (1976) developed the first behavioural treatment of depression, in which patients increased the number of both pleasant activities and positive interactions with their social environment. Several promising trials were conducted but these were forgotten with the emergence of cognitive therapy for depression in the 1980s.

Jacobson et al (1996) set up an important study to assess the value of the components of cognitive therapy. They randomised 150 people with depression to three groups: activity scheduling; activity scheduling plus cognitive challenges to automatic thoughts; and activity scheduling plus cognitive challenges to automatic thoughts, core beliefs and assumptions (full cognitive therapy). They found no statistically or clinically significant differences between the groups and concluded that the cognitive component was redundant. This outcome remained at 2-year followup (Gortner et al, 1998). Subsequent meta-analyses of 17 studies involving over 1000 participants (Cuijpers et al, 2006; Ekers et al, 2007) found no difference in efficacy between behavioural approaches and cognitive therapy in the treatment of depression in adults. Activity scheduling has also been used with success in people with dementia, after the training of their caregivers (Teri et al, 1997), and in psychiatric in-patients with depression (Hopko et al, 2003a).

In a literature review Longmore \& Worrell (2007) found little evidence that challenging the content of thoughts significantly increased the effectiveness of cognitive-behavioural therapy (CBT) and little empirical support for the causative role of cognitive change in the symptomatic improvements achieved in the therapy. The review did not, however, include some of the more recent studies in anxiety disorders, which have found cognitive approaches to enhance graded exposure and response prevention.

\section{Theory and rationale of behavioural activation}

Behavioural activation is a development of activity scheduling, which is a component of cognitive therapy. Introduced by Martell et al (2001), it has two primary focuses: the use of avoided activities as a guide for activity scheduling and functional analysis of cognitive processes that involve avoidance (a glossary of terms appears in Box 1). A simpler version of activity scheduling without a functional analysis of cognitive processes is described by Hopko et al (2003b).

Behavioural activation is grounded in learning theory and contextual functionalism. It is not about scheduling pleasant or satisfying events (as in the first stage of cognitive therapy). It does not focus on an internal cause of depression such as thoughts, inner conflicts or serotonergic dysfunction. The focus is on the whole event and variables that may influence the occurrence of unhelpful responses - both overt behaviour and cognitive processes. Contextualisation

David Veale is an honorary senior lecturer at the Institute of Psychiatry, King's College London and a consultant psychiatrist in cognitivebehavioural therapy at the South London and Maudsley Trust (Centre for Anxiety Disorders and Trauma, The Maudsley Hospital, 99 Denmark Hill, London SE5 8AF, UK. Email: David.Veale@iop.kcl.ac.uk; website: http:/ /www.veale.co.uk) and the Priory Hospital North London. He is currently President of the British Association of Behavioural and Cognitive Psychotherapies. 


\section{Box 1 Glossary of terms}

Activity scheduling In behavioural activation, this a way of structuring one's day according to activities that are avoided and which is consistent with one's valued directions

Behavioural activation An evidence-based treatment for depression by Martell et al (2001). One of the family of behavioural and cognitive psychotherapies

Cognitive fusion Ways in which thoughts, images or associations from the past become fused with reality and guide one's behaviour

Contextual functional analysis Analysis of the function of typical cognitive processes and behaviours: a way of identifying antecedents and consequences of a response, used to determine the factors that maintain depressed mood (see Box 3)

Development formulation Similar to a standard psychiatric formulation, but with the emphasis on the social context - factors such as loss, interpersonal conflict or changes in role - and the way in which these factors have led the patient to cope

Valued direction What is important in one's life. Values are not goals - they are more like a guiding compass and must be lived out by committed action

takes a pragmatic approach, looking at what predicts and maintains an unhelpful response by various reinforcers that prevent the person from reaching their goals.

During their first sessions the rationale behind the therapy is outlined clearly for the patient. The therapist gives positive explanation for the patient's symptoms and seeks feedback to illustrate how the patient's solutions are the problem, maintaining their distress and handicap. For example, a patient might be told that their depression is highly understandable given the context in which they find themselves (perhaps a conflict in a relationship or a significant loss). The experience of depression is regarded as a consequence of avoiding or escaping from aversive thoughts or feelings (called 'experiential avoidance'). It is emphasised that this, too, is an entirely understandable and natural response.

As therapy progresses, patients are taught how to analyse the unintended consequences of their ways of responding, including inactivity and ruminating (e.g. trying to find reasons for the past or attempting to solve insoluble problems). They are shown that the effect of their ways of coping is that they become withdrawn and avoid both their normal activities and social interaction. This in turn leads to deeper depression, more rumination and missing out on experiences in life that normally bring satisfaction or pleasure. Furthermore, the way they act affects their environment and other people in a way that can aggravate the depression.

\section{Assessment and formulation}

A development formulation (Box 2) is made that focuses on social context and the way in which this has shaped the patient's coping behaviours. In each session, the therapist tries to determine what contextual factors are involved in the way the individual is thinking and feeling and how that person responds to whatever factors seem to be maintaining their depressed mood. The key issue in the formulation is determining the nature of the avoidance and escape, and using this to guide the planning of alternative 'approaching' behaviours.

Figure 1 shows the formulation for a 45 -year-old married man who has been made redundant and is avoiding seeking a new job and making any decisions. His depression is explained as a consequence of his avoidance or escaping from thoughts of failure and feelings of shame. Avoidance leads to low levels of positive reinforcement and a narrowing of his normal repertoires. The diagram highlights the various secondary coping strategies that maintain the

\section{Box 2 Examples of avoidance in depression}

Social withdrawal

- Not answering the telephone

- Avoiding friends

Non-social avoidance

- Not taking on challenging tasks

- Sitting around the house

- Spending excessive time in bed

Cognitive avoidance

- Not thinking about relationship problems

- Not making decisions about the future

- Not taking opportunities

- Not being serious about work or education

- Ruminating on trying to explain the past or solve insoluble problems

Avoidance by distraction

- Watching rubbish on television

- Playing computer games

- Gambling

- Comfort-eating

- Excessive exercise

Emotional avoidance

- Use of alcohol and other substances 


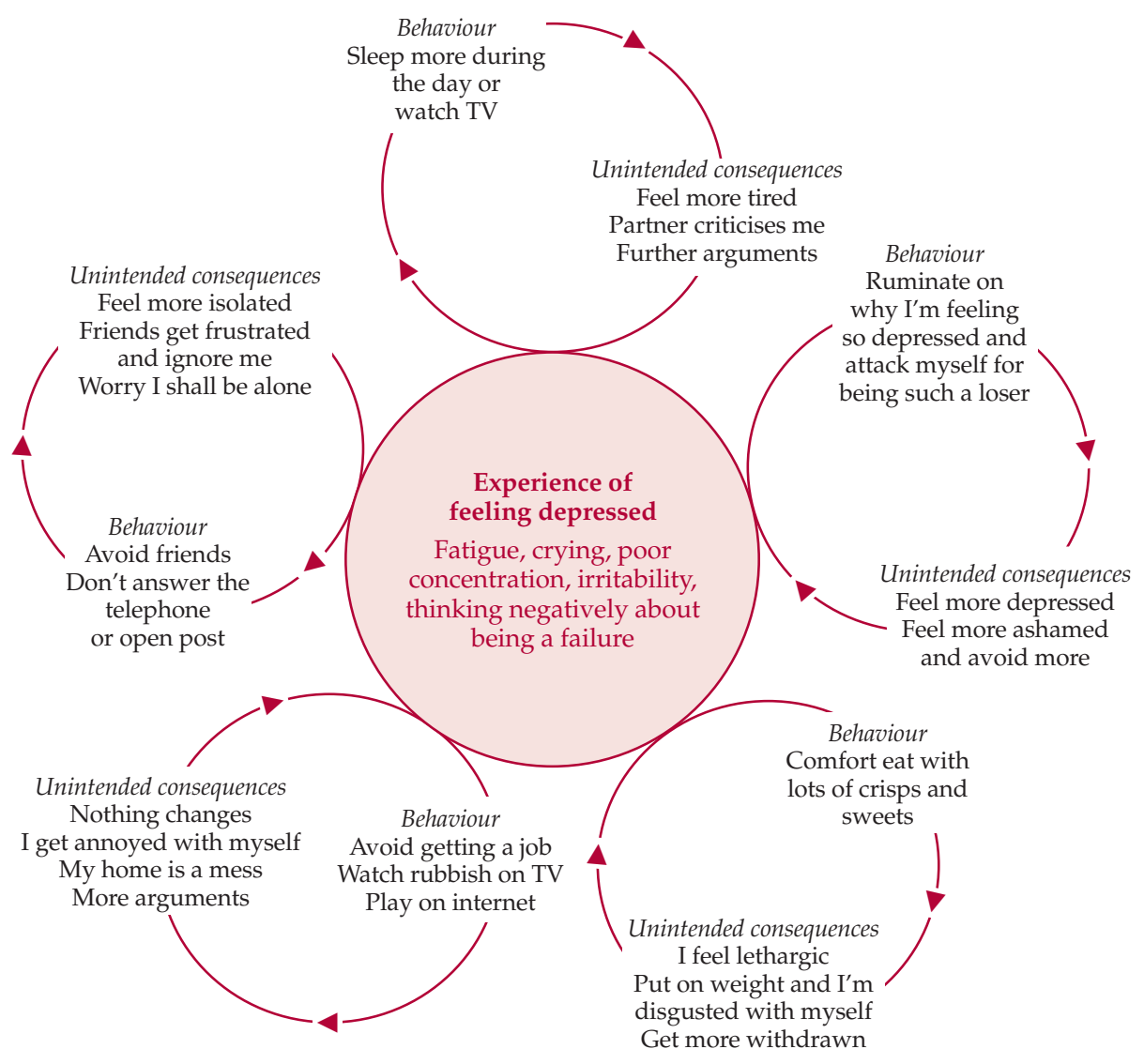

Fig. 1 A formulation of depression in a married man who has been made redundant and is avoiding seeking a new job and making decisions.

experience of being depressed. These appear in the circles surrounding the central, shaded circle, much as petals on a flower. Behavioural activation aims to break off each of these 'petals', to help the individual to use approaching rather than avoiding behaviours and to become active despite their negative feelings or lack of motivation.

Secondary coping behaviours are targeted in all types of depression, but especially when the individual is unaware of precipitating factors or, in chronic depression, when there is no obvious trigger or onset. In individuals who have a biological vulnerability, whose depression may come 'out of the blue' (without any apparent context), the formulation still focuses on their reaction to the experience of being depressed and their escape from aversive thoughts and feelings, which are the immediate reinforcers of their illness.

An activity log may be kept to assess the individual's pattern of responding and the link with alterations in mood. It may also be used to assess the breadth or restriction of activity, which can then be discussed during sessions. Avoidance can take many forms (Box 2) and the Cognitive- Behavioural Avoidance Scale (Ottenbreit \& Dobson, 2004) can help in assessing the degree of avoidance across different domains. Any activity may be subject to a contextual functional analysis, and individuals are taught to conduct their own analysis of their way of coping with a situation to determine whether it is helpful and what is being avoided. They are shown that they do have a choice. If they choose to use avoidance, they should monitor its effect on their mood. They can be encouraged to conduct a behavioural experiment to compare the effect of avoidance or rumination with that of approaching behaviours, perhaps on alternate days, and to record the effect on their mood and distress.

\section{Goals and valued directions}

All patients should have clearly defined goals in the short, medium and long term that are related to their avoidance and can be incorporated into activity scheduling and regularly monitored. Sometimes the goals will compete and then only some of them will be met. Goals should include a return to normal work and social roles as soon as possible. For those who have been out of work for a long period, part-time work in a voluntary capacity or retraining might be appropriate. 
A feature borrowed from acceptance and commitment therapy (Hayes et al, 1999) is to identify the individual's valued directions and what they want their life to stand for. The activity schedule that they draw up can then be focused not only on what they are avoiding but also on what is important to them (although the two often overlap).

The Valued Living Questionnaire (Hayes et al, 1999) is a useful instrument for helping individuals identify their valued directions. It offers prompts for different types of value ('areas'), about which the individual writes a brief statement. Patients should be warned not to follow values simply because others will approve.

Values (valued directions) are not goals - they are more like a compass and must be lived out by committed action. Thus, getting married is a goal, but being a good partner is a value: you never reach your destination as there is always something more you can do. If a valued direction in life is to be a good parent, then the first goal for a depressed patient might be to spend a specified time each day playing, reading or talking with their child.

\section{Structure}

Like standard CBT, a typical behavioural activation session has a structured agenda to review the homework and the progress towards the goals, to discuss feedback on the previous session and to focus on one or two specific issues. The number of sessions to treat depression would be between 12 and 24 . Homework is more likely to be carried out if the individual is actively engaged in setting it and if there are agreed times or places when it will be carried out. Sessions are collaborative and the patient is expected to be active and to try to generate solutions. Like CBT, behavioural activation is not didactic but takes the form of a Socratic dialogue. Sessions are best videoor audio-taped for the patient to listen to again and for therapist supervision.

The context of the relationship with the therapist is important. Techniques of functional analytic psychotherapy (Kohlenberg et al, 2004) introduce learning theory into the therapeutic relationship, showing how it can enhance change towards the goals. It brings the patient's attention to what they are currently thinking, feeling and doing about the therapist and the therapeutic relationship. The therapist identifies behaviours within the session that are examples of the patient's problems and uses their own behaviour to decrease these; likewise, the therapist identifies improvements in the patient's daily life and responds to reinforce these. The effect of the therapist's behaviour on the patient would be observed and the reinforcement adjusted as necessary.

\section{Activity scheduling}

The core of behavioural activation is gradually to identify activities and problems that the individual avoids and to establish valued directions to be followed. These are set out on planned timetables (activity schedules). Individuals are encouraged to start activity scheduling with short-term goals and to treat their timetables as a series of appointments with themselves. A major mistake is for a patient to try to tackle everything at once. The aim is to introduce small changes, building up the level of activity gradually towards long-term goals. Days should not be filled with activity for activity's sake. The activities chosen must relate to what the individual has been avoiding and help them to act in accordance with their valued directions. Individuals are, however, encouraged to include activities that are soothing and pleasurable, as rewards.

Individuals should monitor the effect of their scheduled activities (and deviations from their plan) on their mood. They should also evaluate whether what they did was in keeping with their goals and valued directions. They are encouraged to note, and the therapist should assess, areas that are still avoided and activities that are overused to avoid problematic or painful thoughts and feelings. The therapist might assist with problem-solving or use role-play to practise activities during a session.

\section{What does one do with cognitions?}

In behavioural activation, therapists tend not to become engaged in the content of the patient's thinking. Instead they use functional analysis to focus on the context and process of the individual's response (Box 3). The most common cognitive responses are rumination, fusion and self-attack.

Rumination frequently involves trying to answer questions that cannot be answered, constantly seeking reasons for the depression, fantasising ('If only I'd found a way to make him different') or self-pity ('What have I done to deserve being treated this way?).

Individuals with chronic depression and low self-esteem may attack themselves verbally ('You fat, useless piece of shit') or frequently compare themselves to others.

Both rumination and self-attack serve to avoid aversive situations such as silence or provide escape from thinking about interpersonal problems or feelings. The therapist encourages the individual to be aware of the context (the antecedents) in which these responses occur and the consequences of engaging in them. These consequences usually involve some form of avoidance and non-goal-directed activity. 


\section{Box 3 Contextual functional analysis: the} ABCDE

The following questions might be asked of a patient who believes they are worthless.

Antecedents or context

In what situations in the past have you thought that you were worthless?

Behaviour and cognitive processes in response What do you do next when you think you are worthless? Does your way of responding include a pattern of avoidance (e.g. staying home, not answering the phone, going to bed and ruminating)?

\section{Consequences}

What immediate effect does this activity have? Does it make you feel more comfortable? Does it stop you feeling or thinking something painful?

What unintended effects does this activity have? Does it make you feel more hopeless, tired or depressed? What effect does this activity have on others? Do they get annoyed and critical?

\section{Directions}

What alternative activities could you choose that are in keeping with your goals and valued directions?

\section{Effect}

What effect did following your goal or valued direction have?

Individuals are helped to turn 'Why' or 'If only' questions into 'How' questions that relate to attaining their goals and following their valued directions and that can be incorporated into their activity schedules.

Cognitive fusion describes the way in which thoughts or images from the past become fused with reality, and information about the world is obtained from this revised internal reality. Patients are taught to become more aware of their surroundings and to see events for what they are, rather than what their mind is telling them. This process is akin to mindfulness and involves separating the thought of an event from the experiencing of it. Patients are taught to distance themselves from thoughts and no longer to engage with or 'buy into' them. A metaphor for thoughts and urges that I like is traffic on a road. Engaging with thoughts is akin to standing in the road and trying to divert the cars (and getting run over) or trying to get one and find a parking space for it. However, even if one manages to divert or park one car there are always more to be dealt with. The goal is to acknowledge the thoughts but not to attempt to stop or control or answer back at them. The aim is to accept fully aversive thoughts and to 'walk along the side of the road', engaging with life despite the traffic, which one can quietly ignore (Wells, 2006; Veale, 2007).

\section{Obstacles to activity scheduling}

The most common obstacle to implementing behavioural activation are the individual's beliefs about avoidance: people tell themselves that they will engage in a particular activity when they feel motivated or when they 'feel like it'. The solution is that they should always act according to the plan or activity schedule - not according to how they feel at the time. Individuals are told that the longer they wait, the greater the likelihood that they will become even less motivated: if necessary, the task should be done now, even in an unmotivated way. 'Just doing it' leads to differences in the way the individual thinks and feels, which in turn increases motivation and changes the way others view them.

\section{Other approaches consistent with behavioural activation}

A number of approaches recommended by the National Institute for Health and Clinical Excellence for mild to moderate depression (National Collaborating Centre for Mental Health, 2004) are consistent with behavioural activation and can, if needed, be woven into therapy (Box 4).

\section{Exercise and healthy eating}

Becoming fitter is not important in behavioural activation but increasing activity levels is. A key issue is trying to find an activity that fits the individual's personality, for example their degree of competitiveness or sociability (Veale \& Willson, 2007).

\footnotetext{
Box 4 Approaches complementary to behavioural activation

- Exercise and healthy eating

- Problem-solving therapy

- Sleep management

- Counselling

- Family or couple therapy

- Compassionate mind training

- Acceptance and commitment therapy
} 
People with depression may also eat chaotically, neglect to eat or live off junk food. The function of this may be to numb themselves emotionally. Eating healthily can be incorporated into activity scheduling (e.g. growing and buying food, preparing meals, eating at set times and occasionally eating out).

\section{Problem-solving therapy}

Problem-solving therapy (D'Zurilla \& Nezu, 2006) identifies the problems to be solved and the steps a person might take to try to solve them. Most people do not lack problem-solving skills, but they may be avoiding their problems. This is where the therapy becomes integral to behavioural activation. For example, a woman who is being physically abused by her partner can be given information on a women's shelter and could be encouraged to do a cost-benefit analysis on using it.

Problem-solving should not be used with nonexistent problems or worries of the 'What if' type, as it would merely generate further questions and worry.

\section{Sleep management}

Sleep management (Wilson \& Nutt, 1999) is integral to behavioural activation. Many individuals with depression have a chaotic sleep pattern or use sleep to avoid activity. It is important to do a functional analysis on the pattern of sleep and, if necessary, integrate a sleep routine into an activity schedule.

\section{Counselling}

Counselling is consistent with behavioural activation provided that it is supportive in helping people to move on in their lives and solve their problems. Analytical counselling, trying to find reasons in the past for current problems - 'getting to the bottom of it all' - may be counterproductive and can encourage rumination and further depression.

\section{Family or couples therapy}

Sometimes partners and other family members reinforce an individual's avoidance; they can be overprotective, aggressive or sarcastic; they may minimise the problem or avoid the individual. Different members of the family may use different ways of coping with a depressed relative, leading to further discord on the 'best way'.

Assessment should focus on the way different members of the family cope with the patient's depression, their attitudes to treatment and how their response affects the patient. Behavioural activation might be used to help the family to be consistent and emotionally supportive, perhaps helping the individual to follow their activity schedule.

\section{Compassionate mind training}

Compassionate mind training (CMT; Gilbert, 2005) is a newer development that can be integrated into behavioural activation with individuals who have chronic problems associated with shame, self-criticism or self-attacking. Like behavioural activation, CMT is based on a functional analysis of the self-directed behaviour and teaches individuals to develop selfcompassion and soothing behaviours.

\section{Acceptance and commitment therapy}

Acceptance and commitment therapy (ACT; Hayes et al, 1999) is another newer development within the family of behavioural and cognitive psychotherapies and it has an increasing evidence base. Its principles overlap with those of behavioural activation, and its focus is on the way individuals perpetuate their difficulties through the language they use and on how they can learn to act in a valued direction despite their feelings. There is rich use of metaphors and detached mindfulness of one's thoughts without challenging their content.

\section{In-patients and day patients with depression}

Behavioural activation programmes can be developed for in-patients and day patients with more severe depression but they will depend on the enthusiasm and leadership of a psychiatrist and supervised staff trained in the technique (Rogers et al, 2002a,b). Patients' actual and planned goals and activity levels, their avoidance profile and valued directions might be reviewed by nursing staff in a group or individually at the beginning and end of each day. In their daily interactions with patients, nursing staff would discourage avoidance and reinforce approach behaviours.

Creativity may be required to ensure that individuals act in their valued directions during their in-patient stay. In tackling avoidance of friends, for example, the patient might first be encouraged to make contact by text message, followed up by telephone calls or a meeting and making plans for the future. A valued direction for friendship that is honest and caring might be explored by sharing with a friend what it is like to be depressed and asking about the friend's experience of the depression; this might open up the opportunity to give and receive emotional support. 


\section{Antidepressant medication}

It is more scientifically correct to tell patients that neurotransmitter abnormalities are not causes of depression so much as associations with it, and that an antidepressant may enhance neural transmission rather than correct a defect. Antidepressant medication can be combined with behavioural activation in moderate to severe depression, although no data exist on whether the combination increases efficacy or cost-effectiveness of treatment, especially in the long term.

It can sometimes be helpful to consider identifying the function of psychotropic medication for a patient and whether they are seeking medication to avoid aversive thoughts and feelings. It might be said that the goal of antidepressant medication is to feel better, whereas the goal of behavioural activation is to help the individual both to develop better feelings and to do the things they value in life - including whatever they are avoiding - despite the way they feel. Philosophically, behavioural activation teaches patients that depression is a natural response to an aversive environment, rather than a brain dysfunction, although it does accept that there is a stronger biological component in some forms of depression.

A big problem is patient choice and access to evidence-based psychological therapies: individuals are more likely initially to be offered medication, whereas they would have been more likely to have chosen an evidence-based psychological treatment, had it been offered (Veale, 2008).

\section{The evidence base for behavioural activation}

A key evaluation of behavioural activation as a treatment for depression occurred in a randomised controlled trial in which it was compared with standard CBT, an antidepressant (paroxetine) and a drug placebo in 214 out-patients (Dimidjian et al, 2006). Participants receiving behavioural activation or CBT attended a maximum of $24 \times 50$-minute sessions over 16 weeks. Depression, therapist adherence, therapist competence, response and remission were measured. In the participants with more severe depression, behavioural activation was found to be as efficacious as paroxetine and more efficacious than CBT. Compared with paroxetine, behavioural activation brought a greater percentage of participants to remission and retained a greater percentage in treatment.

Thus, behavioural activation is effective across the spectrum of illness severity, as a low-intensity treatment for mild to moderate depression in the community up to intensive treatment for day patients and in-patients with severe depression. It is therefore a very suitable therapy for use in stepped care. (Stepped care is a way of using limited resources to greatest effect. For most people, complex interventions are given only when simpler and cheaper ones have been shown to be inadequate.) Behavioural activation lends itself to manualised self-help although the long-term cost-effectiveness of self-help books for the intervention (Addis \& Martell, 2004; Veale \& Willson, 2007) with or without the support of a low-intensity worker has yet to be evaluated. Another potentially fruitful possibility would be computerised behavioural activation with minimal support for mild depression.

\section{Why choose behavioural activation?}

Behavioural activation may be currently unpopular because it lacks the complexity of other psychotherapies (e.g. cognitive therapy's challenging of core beliefs and schemas). For some, it has associations of reward and punishment or a therapist who is cold and unresponsive. Some think it a simple therapy suitable only for mild illness. The principles may be relatively simple but it is still hard for the patient to carry out. Furthermore, the therapist must still make an individual formulation of the factors maintaining the patient's depression and must have good supervision and training. Complex problems often require the therapist to carry out simple procedures well rather than undertaking ever more complex ones.

An advantage of behavioural activation over traditional cognitive therapy for depression is that it may be easier to train staff in its use. And as discussed above, it may have greater efficacy in severe depression. Among its advantages over antidepressants is that patients may find it more acceptable and it may be more cost-effective in the long term.

The priority for research is to determine the costeffectiveness of behavioural activation for both mild to moderate depression with low-intensity workers in stepped care and for severe depression either alone or in combination with antidepressant medication.

\section{Declaration of interest}

None.

\section{References}

Addis, M. E. \& Martell, C. R. (2004) Overcoming Depression One Step at a Time. New Harbinger Publications.

Cuijpers, P., van Straten, A. \& Warmerdam, L. (2007) Behavioral activation treatments of depression: a meta-analysis. Clinical Psychology Review, 27, 318-326. 
Dimidjian, S., Hollon, S. D., Dobson, K. S., et al (2006) Randomized trial of behavioral activation, cognitive therapy, and antidepressant medication in the acute treatment of adults with major depression. Journal of Consulting and Clinical Psychology, $74,658-670$

D'Zurilla, T. J. \& Nezu, A. M. (2006) Problem-Solving Therapy: A Positive Approach to Clinical Intervention. Springer Publishing.

Ekers, D., Richards, D. \& Gilbody, S. (2007) A meta-analysis of randomized trials of behavioural treatment of depression. Psychological Medicine, published online ahead of print, doi: 10.1017/S0033291707001614.

Ferster, C. B. (1973) A functional analysis of depression. American Psychologist, 28, 857-870.

Gilbert, P. (ed.) (2005) Compassion: Conceptualisations, Research and Use in Psychotherapy. Brunner-Routledge.

Gortner, E. T., Gollan, J. K., Dobson, K. S, et al (1998) Cognitive behavioral treatment for depression: relapse prevention. Journal of Consulting and Clinical Psychology, 66, 377-384.

Hayes, S. C., Strosahl, K. D. \& Wilson, K. G. (1999) Acceptance and Commitment Therapy. Guilford.

Hopko, D. R., Lejuez, C. W., Lepage, J. P., et al (2003a) A brief behavioral activation treatment for depression; a randomized pilot trial within an inpatient psychiatric hospital. Behavior Modification, 27, 458-469.

Hopko, D. R., Lejuez, C. W., Ruggiero, K. J., et al (2003b) Contemporary behavioural activation treatments for depression: procedures, principles, and progress. Clinical Psychology Review, 23, 699-717.

Jacobson, N. S., Dobson, K. S., Truax, P. A., et al (1996) A component analysis of cognitive-behavioral treatment for depression. Journal of Consulting and Clinical Psychology, 64, 295-304.

Kohlenberg, R. J., Kanter, J. W. \& Bolling, M. (2004) Functional analytic psychotherapy, cognitive therapy, and acceptance. In Mindfulness and Acceptance: Expanding the Cognitive-Behavioral Tradition (eds S. C. Hayes, V. M. Follette \& M. M. Linehan), pp. 96-119. Guilford.

Lewinsohn, P. M., Biglan, A. \& Zeiss, A. S. (1976) Behavioral treatment of depression. In The Behavioral Management of Anxiety, Depression and Pain (ed. P. O. Davidson), pp. 91-146. Brunner/Mazel.

Longmore, R. J. \& Worrell, M. (2007) Do we need to challenge thoughts in cognitive behavior therapy? Clinical Psychology Review, 27, 173-187.

*Martell, C. R., Addis, M. E. \& Jacobson, N. S. (2001) Depression in Context: Strategies for Guided Action. Norton.

National Collaborating Centre for Mental Health (2004) Depression: Management of Depression in Primary and Secondary Care. NICE. http: / / guidance.nice.org.uk/CG23

Ottenbreit, N. D. \& Dobson, K. S. (2004) Avoidance and depression: the construction of the Cognitive-Behavioral Avoidance Scale. Behaviour Research and Therapy, 42, 292-313.

Rogers, P., Curran, J. \& Gournay, K. (2002a) Depression: nature and treatment using behavioural activation (Part One). Mental Health Practice, 5, 32-38.

Rogers, P., Curran, J. \& Gournay, K. (2002b) Depression: nature and treatment using behavioural activation (Part Two). Mental Health Practice, 6, 29-36.

Teri, L., Logsdon, R. G., Uomoto, J., et al (1997) Behavioral treatment of depression in dementia patients: a controlled clinical trial. Journal of Gerontology. Series B, Psychological Sciences and Social Sciences, 52, P159-P166.

Veale, D. (2007) Cognitive-behavioural therapy for obsessivecompulsive disorder. Advances in Psychiatric Treatment, 13, 438-446.

Veale, D. (2008) In debate: Layard's proposals for 10000 more therapists in new psychological treatment centres trades on a naively mechanistic concept of 'mental illness' and would be counterproductive across society. British Journal of Psychiatry, in press.

Veale, D. \& Willson, R. (2007) Manage Your Mood. A Self-help Guide Using Behavioural Activation. Constable \& Robinson.

Wells, A. (2006) Detached mindfulness in cognitive therapy: a metacognitive analysis and ten techniques. Journal of RationalEmotive and Cognitive-Behavior Therapy, 23, 337-355.

Wilson, S. \& Nutt, D. (1999) Treatment of sleep disorders in adults. Advances in Psychiatric Treatment, 5, 11-18.

\section{MCQs}

1 Functional analysis:

a ignores cognitive processes such as rumination

b cannot be used for the therapeutic relationship

c cannot be taught to patients

$\mathrm{d}$ is concerned with challenging the function of an activity

e aims to determine whether an activity is immediately reinforcing and more likely to occur again and the unintended consequences in the long term.

2 Cognitive processes in depression include:

a thought dissociation

$\mathrm{b}$ rumination to find reasons for being depressed

c over-inflated sense of responsibility for harm

d cognitive fission

e self-compassion.

3 Activity scheduling involves:

a scheduling only pleasant and satisfying activities

b scheduling activities that have been avoided

c negative reinforcement

d buzzers to prompt the patient

e starting an activity only when a person feels motivated.

4 Assessment for behavioural activation in depression involves:

a beliefs about the self, world and the future

b types and degree of avoidance

c countertransference

d understanding a detailed developmental history and the causes of depression

e ignoring the context of interpersonal relationships.

5 Behavioural activation:

a should be used only for simple cases of depression

$\mathrm{b}$ is linked to operant conditioning of rewards and punishments

c has, for some, an incorrect association of reward and punishment or a cold, unresponsive therapist

$\mathrm{d}$ is a preliminary to cognitive or 'deeper' therapy

e can be used in people with severe depression as an alternative to antidepressants. 